\title{
Most canine ameloblastomas harbor HRAS mutations, providing a novel large-animal model of RAS-driven cancer
}

\author{
Persiana S. Saffari ${ }^{1}$, Natalia Vapniarsky², Anna S. Pollack', Xue Gong ${ }^{1}$, Sujay Vennam¹, Andrew J. Pollack' ,
} Frank J. M. Verstraete ${ }^{3}$, Robert B. West ${ }^{1}$, Boaz Arzi ${ }^{3}$ and Jonathan R. Pollack ${ }^{1}$

\begin{abstract}
Canine acanthomatous ameloblastomas (CAA), analogs of human ameloblastoma, are oral tumors of odontogenic origin for which the genetic drivers have remained undefined. By whole-exome sequencing, we have now discovered recurrent HRAS and BRAF activating mutations, respectively, in 63\% and 8\% of CAA. Notably, cell lines derived from CAA with HRAS mutation exhibit marked sensitivity to MAP kinase (MAPK) pathway inhibitors, which constrain cell proliferation and drive ameloblast differentiation. Our findings newly identify a large-animal spontaneous cancer model to study the progression and treatment of RAS-driven cancer. More broadly, our study highlights the translational potential of canine cancer genome sequencing to benefit both humans and their companion animals.
\end{abstract}

\section{Introduction}

As do humans, domestic dogs develop spontaneous cancers with genetic and environmental influences ${ }^{1,2}$. Common cancers in dogs include lymphoma, osteosarcoma, mammary carcinoma, hemangiosarcoma, oral melanoma, and mast cell tumors, among others. Canine cancers display strong similarities to their human counterparts in histopathology, tumor genetics, and clinical behavior. With millions of pet dogs cared for into old age (and about half developing cancer), dogs offer a largely untapped resource for new cancer insight, as well as advantageous models for preclinical testing ${ }^{3}$. Toward this end, and enabled by the completion of the canine

Correspondence: Natalia Vapniarsky (vapniarsky@ucdavis.edu) or

Robert B. West (rbwest@stanford.edu) or

Jonathan R. Pollack (pollack1@stanford.edu)

'Department of Pathology, Stanford University School of Medicine, Stanford, CA, USA

${ }^{2}$ Department of Pathology, Microbiology \& Immunology, UC Davis School of Veterinary Medicine, Davis, CA, USA

Full list of author information is available at the end of the article.

These authors contributed equally: Persiana Saffari, Natalia Vapniarsky, Anna S. Pollack reference genome ${ }^{4}$, incipient efforts are underway to systematically sequence canine cancer genomes ${ }^{5-7}$.

Canine acanthomatous ameloblastomas (CAAs) are odontogenic tumors of the jaw, thought to represent the counterpart of human ameloblastoma (acanthomatous histologic variant $)^{8}$. CAAs share with human ameloblastoma their histology, propensity to infiltrate bone while rarely metastasizing, and presumptive origin from the ameloblast (enamel secreting) cell lineage ${ }^{9}$, though non-odontogenic origins have also been speculated. CAAs are found across diverse dog breeds and notably occur far more commonly than do human ameloblastomas ${ }^{10}$. Current recommended treatment of CAA is surgical excision. While human ameloblastomas harbor driver mutations in the mitogen-activated protein kinase (MAPK) pathway (including BRAF, KRAS, NRAS, HRAS and FGFR4) and Hedgehog pathway $(S M O)^{11,12}$, the drivers of CAA have not been known.

\section{Results \\ Frequent HRAS mutations in CAA}

To identify cancer-driving mutations in CAA, we carried out whole-exome sequencing (WES) of formalin-

\section{(-) The Author(s) 2019}

(c) (i) Open Access This article is licensed under a Creative Commons Attribution 4.0 International License, which permits use, sharing, adaptation, distribution and reproduction cc) in any medium or format, as long as you give appropriate credit to the original author(s) and the source, provide a link to the Creative Commons license, and indicate if changes were made. The images or other third party material in this article are included in the article's Creative Commons license, unless indicated otherwise in a credit line to the material. If material is not included in the article's Creative Commons license and your intended use is not permitted by statutory regulation or exceeds the permitted use, you will need to obtain permission directly from the copyright holder. To view a copy of this license, visit http://creativecommons.org/licenses/by/4.0/. 


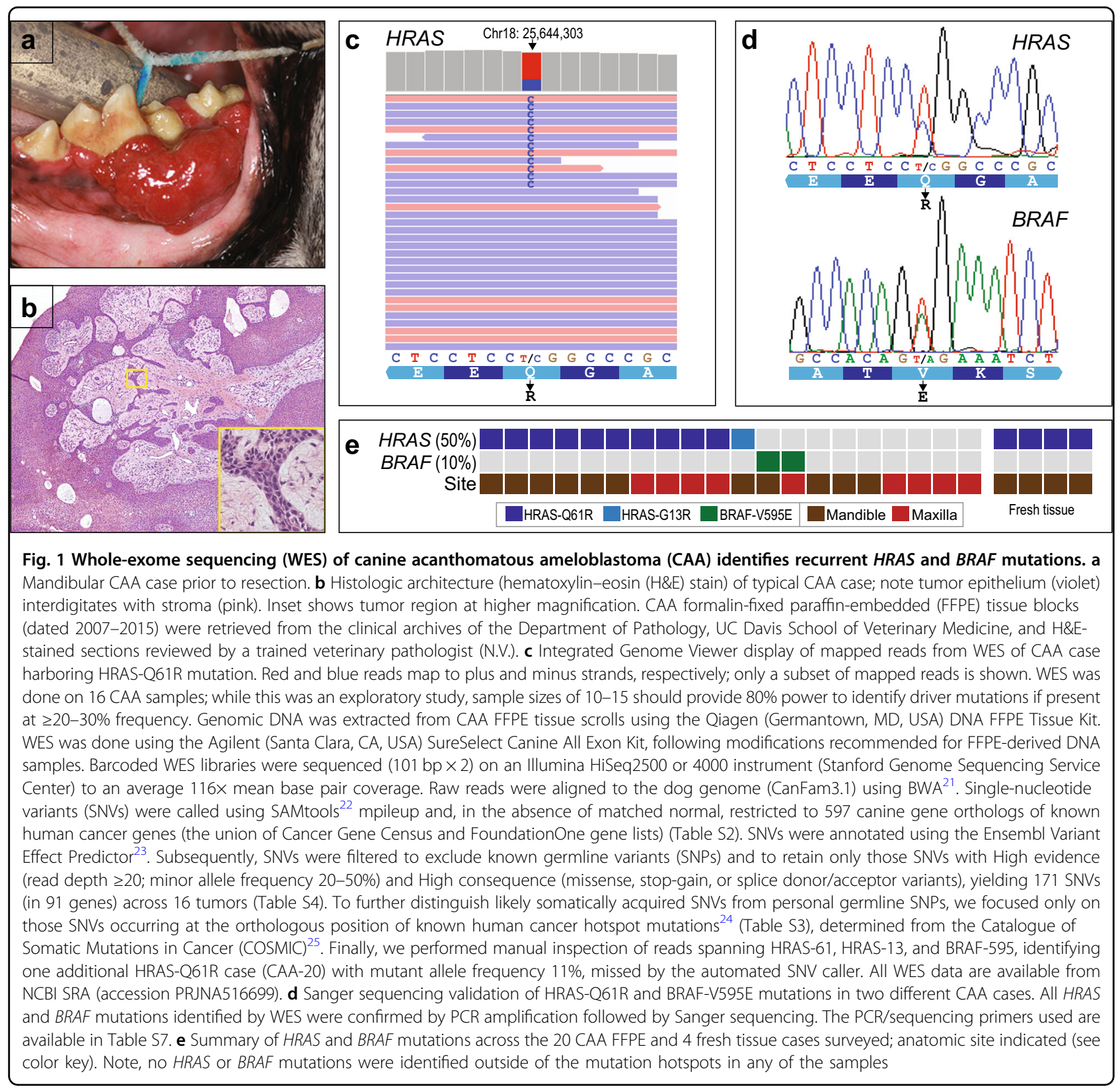

fixed paraffin-embedded (FFPE) tumor tissue from 16 prototypical CAA cases from diverse breeds (Fig. 1a, b and Tables 1, S1). We then used PCR/Sanger sequencing to confirm select mutations in the discovery set plus additional specimens (together totaling 20 CAA cases). Because we lacked matched normal tissue (useful to exclude personal germline single-nucleotide variants (SNVs)), our analysis focused on the canine orthologs of $\sim 600$ known human cancer genes and, within that set, known mutation "hotspot" sites (Fig. S1 and Tables S2, S3).

Strikingly, 11 of the 20 (55\%) CAA cases carried activating HRAS mutations (10 HRAS-Q61R and 1 HRAS-
G13R), and 2 of the $20(10 \%)$ carried activating $B R A F$ mutations (BRAF-V595E, orthologous to the human BRAF-V600E driver mutation) (Fig. 1c-e and Tables 1, S4). In the seven remaining CAA cases, no driver hotspot mutation was identified. HRAS and BRAF mutation allele frequencies (range 11-46\%; mean 29\%) were consistent with somatically acquired mutations (i.e., admixed with normal stroma), which we confirmed in three CAA cases by laser microdissection (and PCR/Sanger sequencing) of separate tumor epithelium and stroma (Fig. S2). In distinction from human ameloblastomas, where $B R A F$ and $S M O$ mutations are preferentially localized, respectively, to mandibular and maxillary tumors ${ }^{12}$, the canine HRAS 
Table 1 Canine acanthomatous ameloblastoma case characteristics

\begin{tabular}{|c|c|c|c|c|c|c|c|c|c|}
\hline & Case ID & Location & Breed & Age (years) & Sex & Weight (kg) & Mutation & Read depth ${ }^{a}$ & VAF \\
\hline \multirow[t]{20}{*}{ FFPE tissue cases } & CAA-01 & Mandible & German Shepherd & 8 & $M C$ & 42 & & & \\
\hline & CAA-02 & Mandible & Labrador Retriever & 13 & FS & 31 & & & \\
\hline & CAA-03 & Mandible & Labrador Retriever & 11 & FS & 33 & HRAS-Q61R & 72 & 0.278 \\
\hline & CAA-04 & Mandible & Pit Bull Terrier & 10 & FS & 27 & HRAS-Q61R & & \\
\hline & CAA-05 & Mandible & Shetland Sheepdog & 11 & $M C$ & 22 & BRAF-V595E & 197 & 0.289 \\
\hline & CAA-06 & Mandible & Border Collie & 8 & $M C$ & 29 & HRAS-Q61R & 63 & 0.270 \\
\hline & CAA-07 & Mandible & Australian Shepherd & 9 & $M C$ & 31 & HRAS-G13R & 38 & 0.421 \\
\hline & CAA-08 & Mandible & Basset Hound & 15 & $M C$ & 36 & HRAS-Q61R & & \\
\hline & CAA-09 & Mandible & Cocker Spaniel & 9 & FS & 15 & HRAS-Q61R & 95 & 0.263 \\
\hline & CAA-10 & Mandible & Husky mix & 10 & FS & 39 & & & \\
\hline & CAA-11 & Mandible & Chesapeake Bay Retriever & 6 & $M C$ & 33 & HRAS-Q61R & & \\
\hline & CAA-12 & Maxilla & Samoyed & 10 & FS & 36 & HRAS-Q61R & 63 & 0.238 \\
\hline & CAA-13 & Maxilla & Beagle & 10 & $M C$ & 12 & & & \\
\hline & CAA-14 & Maxilla & Collie & 11 & $M C$ & 30 & & & \\
\hline & CAA-15 & Maxilla & Labrador Retriever & 12 & $M C$ & 39 & BRAF-V595E & & \\
\hline & CAA-16 & Maxilla & Collie & 11 & $M C$ & 31 & & & \\
\hline & CAA-17 & Maxilla & Standard Poodle & 7 & $M C$ & 25 & & & \\
\hline & CAA-18 & Maxilla & Labrador Retriever & 11 & FS & 26 & HRAS-Q61R & 90 & 0.267 \\
\hline & CAA-19 & Maxilla & English Bulldog & 10 & $M C$ & NA & HRAS-Q61R & 57 & 0.456 \\
\hline & CAA-20 & Maxilla & Beagle mix & 5 & $M C$ & 17 & HRAS-Q61R & 101 & 0.109 \\
\hline \multirow[t]{4}{*}{ Fresh tissue cases } & CAA-21 & Mandible & Labrador Retriever & 8 & FS & 31 & HRAS-Q61R & & \\
\hline & CAA-22 & Mandible & Terrier mix & 8 & FS & 22 & HRAS-Q61R & & \\
\hline & CAA-23 & Mandible & Great Dane & 9 & FS & 53 & HRAS-Q61R & & \\
\hline & CAA-24 & Mandible & Standard Poodle & 3 & $M C$ & 40 & HRAS-Q61R & & \\
\hline
\end{tabular}

$M C$ male castrated, FFPE formalin-fixed paraffin-embedded, FS female spayed, VAF variant allele frequency

${ }^{\mathrm{a}}$ Read depth at mutated base

and BRAF mutations occurred in both anatomic sites (Fig. $1 \mathrm{e})$, and no canine $S M O$ mutations were identified. We also used the WES reads to infer DNA copy number alterations (CNAs); all but one CAA case exhibited relatively flat CNA profiles (Fig. S3).

\section{HRAS mutations confer sensitivity to MAPK pathway inhibition}

To further investigate MAPK pathway-driven CAA, we generated immortalized cell lines from fresh tissue of four additional CAA cases, by conditional reprogramming (i.e., culturing cells with Rho-associated protein kinase (ROCK) inhibitor and irradiated fibroblast conditioned media $)^{13}$. All four cell lines harbored the HRAS-Q61R activating mutation (Fig. 2a, b and Table 1). Testing two of the CAA (HRAS-Q61R) cell lines, both were highly sensitive (at low nanomolar concentrations) to mitogen- activated extracellular signal-regulated kinase (MEK) inhibition by GDC-0623, an allosteric MEK inhibitor that also blocks feedback-mediated RAF/MEK activation ${ }^{14}$ (Fig. 2c). Inhibition of canine MEK activity was confirmed by phospho-extracellular signal-regulated kinase (phospho-ERK) western blot (Fig. 2d). To exclude nonspecific cell toxicity of GDC-0623, we tested CAMA-1 breast cancer cells, which we found consistent with published reports ${ }^{15}$ to be insensitive to MEK inhibition (Fig. 2c). CAA (HRAS-Q61R) cells were also highly sensitive to the MEK inhibitor cobimetinib (GDC-0973), though it has been reported less effective against mutant-RAS than mutant-BRAF-driven tumor models ${ }^{14}$, as well as the ERK inhibitor SCH772984, reported effective against RASdriven cancer models ${ }^{16}$ (Fig. 2c).

Interestingly, MEK inhibition not only blocked CAA (HRAS-Q61R) cell proliferation but also led to cell 


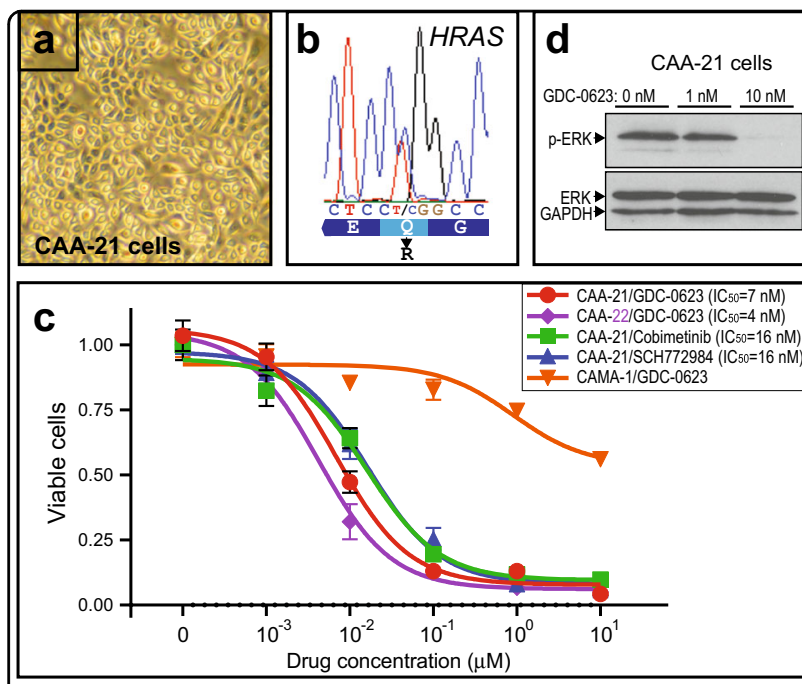

Fig. 2 Canine acanthomatous ameloblastoma (CAA)-derived cell lines harbor HRAS mutation and are sensitive to select mitogenactivated protein kinase (MAPK) pathway inhibitors. a CAA cell line (CAA-21) generated by conditional reprogramming. Fresh CAA tissue (dated 2017-2018) was obtained from tumors excised as part of standard surgical treatment; use of surplus tumor tissue was exempt from IACUC approval. CAA cell lines (Table S1) were established by conditional reprogramming following published methods ${ }^{26}$. Briefly, fresh tumor tissue was minced, and then cells were disaggregated by Collagenase/Hyaluronidase using StemCell Technologies (Vancouver, BC, Canada) reagents and protocols, followed by Trypsin and Dispase. Cells were then filtered through a $40-\mu \mathrm{M}$ cell strainer and plated in Complete F medium (conditioned by irradiated J2 strain mouse Swiss$3 \mathrm{~T} 3$ fibroblasts and containing $10 \mu \mathrm{M}$ ROCK inhibitor (Y-27632)). Cells were passaged by trypsinization, with no appreciable change in growth properties over $>20$ passages. Cell lines are available from J.R. P. upon request. b CAA cells retain HRAS-Q61R mutation, verified by PCR/Sanger sequencing. c MAPK inhibitor dose-response (inhibition) curves depict sensitivity to select MAPK inhibitors. $I C_{50}$ values are indicated. MAPK inhibitors were obtained from Selleckchem (Houston, TX, USA). Drug testing was performed in complete F media (including Y-27632). 50K cells were plated per 6-well plate well (in duplicate) and then challenged with a 10 -fold drug dilution series (or vehicle alone) for $72 \mathrm{~h}$, with daily media/drug replacement. Cell viability was then measured by flow cytometry (BD Accuri C6) or Countess automated cell counter (Thermo Fisher, Waltham, MA, USA). IC 50 values were calculated from dose-response (inhibition) curves using GraphPad Prism. All cell culture experiments were repeated at least twice with comparable results. CAMA-1 cells were obtained directly from the ATCC (Manassas, VA, USA) and cultured as recommended. $\mathbf{d}$ Verification of mitogen-activated extracellular signal-regulated kinase (ERK) inhibitor (GDC-0623) on-target activity in CAA cells; western blot indicates $I_{50}$ (phospho-ERK levels) at 1-10 nM. Western blots were done using primary antibodies against phospho-Erk1/2 (clone D13.14.4E) and Erk1/2 (clone 3A7) (Cell Signaling Technology, Danvers, $M A)$, with detection by chemiluminescence and quantification by ImageJ

flattening reminiscent of cellular senescence and/or terminal differentiation (Fig. 3a, b). To further investigate, we profiled gene expression following MEK inhibition by GDC-0623 (vs. vehicle control). Notably, the genes upregulated by MEK inhibition were significantly

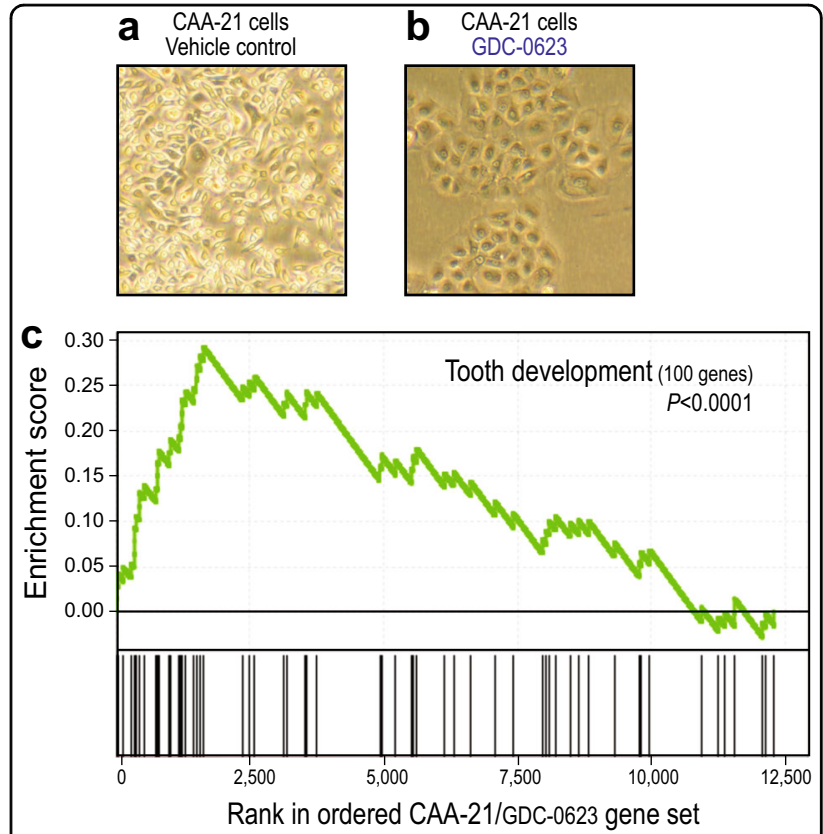

Fig. 3 Transcriptome changes in canine acanthomatous ameloblastoma (CAA) cells induced by mitogen-activated extracellular signal-regulated kinase (MEK) inhibition. a Morphology of CAA cells treated with MEK inhibitor GDC-0623 (vs. vehicle control). b MEK inhibitor addition to CAA (HRAS-Q61R) cells generates a transcriptional response significantly enriched for tooth development genes. c Gene Set Enrichment Analysis (GSEA) enrichment score $P$ value is indicated. Tooth development genes within the leading edge are listed in Table S5. For transcriptome sequencing, CAA-21 cells were plated in 6-well plate wells, and $1 \mu \mathrm{M}$ GDC-0623 (or vehicle control) was added with daily media replacement for $72 \mathrm{~h}$. RNA was isolated using the Qiagen RNeasy Kit, RNAseq libraries generated using Illumina (San Diego, CA, USA) TruSeq RNA Library Prep Kit V2, and barcoded RNAseq libraries sequenced $(101 \mathrm{bp} \times 2)$ on an Illumina HiSeq2500 to an average depth of 20 million reads. Reads were mapped to the Ensembl-annotated (CanFam3.1) transcriptome using TopHat and Cufflinks ${ }^{27}$, and transcripts quantified as Reads Per Kilobase of transcript per Million mapped reads (RPKMs). Enrichment of tooth development genes ${ }^{17}$ was evaluated by GSEA ${ }^{28}$. All RNAseq data are available from NCBI SRA (accession PRJNA516699)

enriched for tooth development genes ${ }^{17}(P<0.0001$; Gene Set Enrichment Analysis) (Fig. 3c and Table S5), supporting an odontogenic origin for CAA. Among these, the ameloblast-specific gene AMTN (Amelotin) ${ }^{18}$ was upregulated $\sim 5000$-fold (Table S6).

\section{Discussion}

Here, by WES of CAA FFPE and subsequent fresh tissue specimens, we have in total identified HRAS activating mutations in $63 \%$ of cases (15 of 24) and BRAF activating mutations in $8 \%$ of cases ( 2 of 24 ). Together, over two thirds $(71 \%)$ of CAA cases carry activating MAPK pathway mutations that should be targetable by existing Food and Drug Administration-approved or investigational 
drugs. Indeed, we demonstrate that CAA cells carrying HRAS-Q61R mutation are highly sensitive to MEK and ERK inhibition. Interestingly, MEK inhibition not only constrains cell proliferation but also appears to drive ameloblast differentiation, noted by the 5000 -fold induction of the ameloblast-specific AMTN transcript.

While most CAA cases harbored HRAS or BRAF mutations, $29 \%$ (7 of 24) carried neither. Because we did not have matching normal DNA (helpful in distinguishing somatic mutations from personal germline variants), we limited our analysis to the canine orthologs of known human cancer gene hotspot mutations. Future studies that include matching normal DNA may reveal additional CAA-driver mutations, either within or outside the MAPK pathway, and should inform mutational burdens as well as signatures suggestive of particular mutational processes.

Additionally, while CAA cells with HRAS-Q61R showed sensitivity to MEK and ERK inhibitors, it remains to be determined whether single-agent therapies will be effective in vivo. For example, with human BRAF-mutant melanomas treated by BRAF inhibition, acquired resistance often develops, while dual BRAF and MEK inhibition has shown improved efficacy ${ }^{19}$.

Importantly, our findings newly identify a large-animal spontaneous tumor model of RAS/RAF-driven cancer, valuable for preclinical testing of MAPK pathway inhibitors. CAA could model MAPK pathway dependence, inhibitor sensitivity, and resistance not only for human ameloblastoma but potentially also for other RAS/RAF mutation-driven human cancers (e.g., thyroid cancer, lung cancer, pancreatic cancer, and melanoma). Surgical excision remains the mainstay treatment of human ameloblastoma, though targeted therapies (particularly MAPK pathway inhibitors) show promise ${ }^{20}$, and regimens might be optimized through preclinical testing in dogs. Our findings also offer more immediate translation in the management of CAA, for example, for compassionate use of MEK/ERK inhibitors in pet dogs that are not surgical candidates (e.g., because of tumor location, extent, or comorbidities). More broadly, our study demonstrates the feasibility, importance, and promise of dog genome sequencing and comparative oncogenomics studies and the commensal benefit to both humans and their companion animals.

\section{Acknowledgements}

We thank the Stanford Genome Sequencing Service Center for providing Illumina sequencing services. This study was supported in part by an NIDCR grant (R01 DE026502) to J.R.P. and R.B.W.

\section{Authors' contributions}

F.J.M.V., R.B.W., B.A., and J.R.P. conceived and planned the studies; P.S., N.V.A., A. S.P., and C.Z. performed experiments; X.G., S.V., and A.J.P. analyzed data; P.S. and J.R.P. drafted the manuscript.

\section{Author details}

${ }^{1}$ Department of Pathology, Stanford University School of Medicine, Stanford, CA, USA. ${ }^{2}$ Department of Pathology, Microbiology \& Immunology, UC Davis School of Veterinary Medicine, Davis, CA, USA. ${ }^{3}$ Department of Surgical \& Radiological Sciences, UC Davis School of Veterinary Medicine, Davis, CA, USA

Conflict of interest

The authors declare that they have no conflict of interest.

\section{Publisher's note}

Springer Nature remains neutral with regard to jurisdictional claims in published maps and institutional affiliations.

Supplementary Information accompanies this paper at (https://doi.org/ 10.1038/s41389-019-0119-1).

Received: 29 December 2018 Accepted: 14 January 2019

Published online: 11 February 2019

\section{References}

1. Khanna, C. et al. The dog as a cancer model. Nat. Biotechnol. 24, 1065-1066 (2006).

2. Schiffman, J. D. \& Breen, M. Comparative oncology: what dogs and other species can teach us about humans with cancer. Philos. Trans. R. Soc. Lond. B Biol. Sci. 370, 20140231 (2015).

3. Kol, A. et al. Companion animals: translational scientist's new best friends. Sci. Transl. Med. 7, 308ps321 (2015).

4. Lindblad-Toh, K. et al. Genome sequence, comparative analysis and haplotype structure of the domestic dog. Nature 438, 803-819 (2005).

5. Sakthikumar, S. et al. SETD2 is recurrently mutated in whole-exome sequenced canine osteosarcoma. Cancer Res. 78, 3421-3431 (2018).

6. Elvers, I. et al. Exome sequencing of lymphomas from three dog breeds reveals somatic mutation patterns reflecting genetic background. Genome Res. 25, 1634-1645 (2015)

7. Harper, K. Canine research to benefit humans and dogs alike. Cancer Discov. 8, 376-377 (2018).

8. Gardner, D. G. Canine acanthomatous epulis. The only common spontaneous ameloblastoma in animals. Oral Surg. Oral Med. Oral Pathol. Oral Radiol. Endod. 79, 612-615 (1995)

9. Yuasa, Y. et al. Amelogenin expression in canine oral tissues and lesions. J. Comp. Pathol. 119, 15-25 (1998).

10. Fiani, N., Verstraete, F. J., Kass, P. H. \& Cox, D. P. Clinicopathologic characterization of odontogenic tumors and focal fibrous hyperplasia in dogs: 152 cases (1995-2005). J. Am. Vet. Med. Assoc. 238, 495-500 (2011).

11. Brown, N. A. et al. Activating FGFR2-RAS-BRAF mutations in ameloblastoma. Clin. Cancer Res. 20, 5517-5526 (2014).

12. Sweeney, R. T. et al. Identification of recurrent $S M O$ and BRAF mutations in ameloblastomas. Nat. Genet. 46, 722-725 (2014).

13. Liu, X. et al. ROCK inhibitor and feeder cells induce the conditional reprogramming of epithelial cells. Am. J. Pathol. 180, 599-607 (2012).

14. Hatzivassiliou, G. et al. Mechanism of MEK inhibition determines efficacy in mutant KRAS- versus BRAF-driven cancers. Nature 501, 232-236 (2013).

15. Barretina, J. et al. The Cancer Cell Line Encyclopedia enables predictive modelling of anticancer drug sensitivity. Nature 483, 603-607 (2012).

16. Morris, E. J. et al. Discovery of a novel ERK inhibitor with activity in models of acquired resistance to BRAF and MEK inhibitors. Cancer Discov. 3, 742-750 (2013).

17. Pemberton, T. J. et al. Identification of novel genes expressed during mouse tooth development by microarray gene expression analysis. Dev. Dyn. 236, 2245-2257 (2007).

18. Iwasaki, K. et al. Amelotin-a novel secreted, ameloblast-specific protein. J. Dent. Res. 84, 1127-1132 (2005).

19. Robert, C. et al. Improved overall survival in melanoma with combined dabrafenib and trametinib. N. Engl. J. Med. 372, 30-39 (2015).

20. McClary, A. C. et al. Ameloblastoma: a clinical review and trends in management. Eur. Arch. Otorhinolaryngol. 273, 1649-1661 (2016). 
21. Li, H. \& Durbin, R. Fast and accurate short read alignment with BurrowsWheeler transform. Bioinformatics 25, 1754-1760 (2009).

22. $\mathrm{Li}, \mathrm{H}$. et al. The Sequence Alignment/Map format and SAMtools. Bioinformatics 25, 2078-2079 (2009)

23. McLaren, W. et al. The Ensembl Variant Effect Predictor. Genome Biol. 17, 122 (2016).

24. Vogelstein, B. et al. Cancer genome landscapes. Science 339, 1546-1558 (2013).

25. Forbes, S. A. et al. COSMIC (the Catalogue of Somatic Mutations in Cancer): a resource to investigate acquired mutations in human cancer. Nucleic Acids Res. 38, D652-D657 (2010)
26. Liu, X. et al. Conditional reprogramming and long-term expansion of normal and tumor cells from human biospecimens. Nat. Protoc. 12 439-451 (2017).

27. Trapnell, $C$. et al. Differential gene and transcript expression analysis of RNA-seq experiments with TopHat and Cufflinks. Nat. Protoc. 7, 562-578 (2012).

28. Subramanian, A. et al. Gene set enrichment analysis: a knowledge-based approach for interpreting genome-wide expression profiles. Proc. Natl. Acad. Sci. USA 102, 15545-15550 (2005). 